\title{
AN ENERGY-EFFICIENT OPTIMIZATION METHOD OF TRAIN GROUP TRAJECTORY FOR METRO
}

\author{
MO CHEN, QINGYUAN WANG, PENGFEI SUN, XIAOWEN WANG \& QIAN FANG \\ Southwest Jiaotong University, China
}

\begin{abstract}
Energy-efficient train operation plays an important role on reduction of energy consumption and sustainable development in Metro system. The improvement of regenerative energy (RE) utilization through multi-train collaborative optimization is an effective way. However, traditional researches on this problem mainly focus on a two-train system, which cannot be applied to train group. This paper proposes a novel optimization method for multi-train, the complex train group problem is turned into a single-train and multiple two-train problems based on the analysis of the total energy model. Then the optimal traction force of the accelerating train related to the braking power of the braking train is deduced to $100 \%$ recover the RE. Therefore, the train group can be optimized by departure orders, traction energy of the first train is minimized and speed profiles of rest trains are adjusted to maximize the utilization of RE by sequence. Specially, optimization of each train is independent, which only needs to focus on the braking power of its previous train, greatly simplifying the multi-train collaborative optimization problem. Detailed optimization methods are proposed and the effectiveness are verified by the simulation results based on Guangzhou Metro.

Keywords: Energy efficient; Metro system; RE; Train group; Collaborative optimization
\end{abstract}

\section{INTRODUCTION}

Metro system has developed rapidly in recent years due to its large capacity, high security and reliable service [1], however, huge energy consumption and $\mathrm{CO}_{2}$ emissions deserve attention, it's urgent to cut down the operational cost. Energy-efficient train control and scheduling are considered two effective ways in the reduction of energy consumption, which are low cost and easy to implement, the former focuses on the control strategy of trains and the latter focuses on the timetable. Besides, reducing energy consumption can also be divided into two aspects, reducing the traction energy and improving the utilization of regenerative energy (RE).

Reduction of the traction energy only relates to the energy-efficient train control of singletrain, purpose of which is to find a sequence of control strategies between two substations with a fixed trip time, and the optimal sequence with maximum traction (MT), cruising (CR), coasting (CO) and maximum braking (MB) for a general line considering the speed limit was theoretically proved by Howlett with using Kuhn-Tucher equation [2]. Then the constraint of continuously-varying gradients was considered in [3]. [4]-[6] delved into the optimal strategy with comprehensive consideration of various operation constraints. Research on reduction of the traction energy has matured, the optimal speed profile to minimize the traction energy of single-train can be easily obtained, however, trajectory optimization of single-train didn't take the energy interaction between trains into account, the utilization of RE plays a significant role in realistic operation of Metro system, which can further reduce the total energy consumption and becomes a hot point in recent years.

Improvement of the utilization of RE relates to both scheduling and energy-efficient train control of multi-train. Designing the timetable with fixed control strategy was proved to be an effective way to reduce the total energy compared to single-train optimization, common approaches include distributing the trip time of each subsection for the entire route [7], 
increasing the overlapping time between the accelerating time and the braking time of adjacent trains [8]. A full review of the study on timetable optimization was given by [9]. Energy saving effect by scheduling is still limited, as a consequence, control strategies collaborative optimization of multi-train with fixed timetable is getting more attention. Speed profiles of trains was optimized to maximize the utilization of RE in one power supply segment by using numerical algorithm [10]. [11] adjusted the mode of train movement in real-time based on an electrical model without meeting the punctuality needs. Tang [12] presented the quadratic programming (QP) model of multi-train operation problem, but the model was too difficult. Liu [13] optimized the tracking train with four and five movement control strategy and compared the energy saving effect. Chen [14] proposed that seven movement strategy has better performance than five movements strategy in big downhill for multi-train and evaluated the transmission losses of RE on the catenary. Although the trajectory optimization of multi-train in above literatures achieved comfortable results, the solution methods are limited to a twotrain system, the collaborative optimization of train group trajectory is more beneficial to the operation in practical Metro system, which is a complex problem and needs to be addressed urgently.

In this paper, a novel trajectory optimization approach of train group is proposed for maximizing the utilization of RE and reducing the total energy. The complex collaborative operation problem of train group is turned into a single-train and multiple two-train problems by analysing the total energy model. Then an optimization method is proposed to make full use of RE in a two-train system, thus making energy interaction only happen between adjacent trains and the optimization of each train becomes independent, therefore, the trajectory of train group can be optimized by departure orders. Simulation results based on Guangzhou Metro Line 8 verify the effectiveness of proposed methods and achieve comfortable energy saving effect.

\section{MATHEMATICAL MODEL}

\subsection{Train Movement Model}

The motion of a train can be described as following dynamic system.

$$
\begin{gathered}
\frac{d v}{d x}=\frac{F_{t}(v)-F_{b}(v)-R_{1}(v)-R_{2}(x)}{v} . \\
\frac{d t}{d x}=\frac{1}{v} .
\end{gathered}
$$

where $v, x$ and $t$ are the speed, position and running time of the train, respectively. $F_{t}(v)$ represents the traction force and $F_{b}(v)$ represents the braking force. $R_{1}(v)$ is the basic running resistance which is defined as follow:

$$
R_{1}(v)=c_{0}+c_{1} v+c_{2} v^{2}
$$

here, the $c_{0}, c_{1}$ and $c_{2}$ are the basic resistance coefficient, which are non-negative constants for a special vehicle type. 
$R_{2}(x)$ is the additional resistance and can be described as follow:

$$
R_{2}(x)=\gamma+M g \frac{A}{r} .
$$

where the first item represents the gradient resistance and the second item represents the curve resistance, $\gamma$ is the gradient at position $x$, which is positive for uphill and negative for downhill, $M$ denotes the train total mass, $g$ represents the gravitational acceleration, $r$ is the radius of the curve at position $x$, and $A$ is a constant.

Several constraints must be satisfied during the operation process of a train, assume the train runs in the subsection which starts at position $S_{1}$ and ends at position $S_{2}$ with the planned trip time $T$, the boundary conditions are:

$$
\begin{gathered}
v\left(S_{1}\right)=0 \quad v\left(S_{2}\right)=0 . \\
t\left(S_{2}\right)-t\left(S_{1}\right)=T .
\end{gathered}
$$

Besides, the speed of train should not exceed the limit for safety, the traction force and the braking force are taken from 0 to the maximum, which are regarded as continuous variables and shown as follow:

$$
\begin{gathered}
0 \leq v(x) \leq V(x) . \\
0 \leq F_{t}(v) \leq F_{t}^{\max }(v) . \\
0 \leq F_{b}(v) \leq F_{b}^{\max }(v) .
\end{gathered}
$$

here, $V(x)$ is the speed limit at position $x, F_{t}^{\max }$ and $F_{b}^{\max }$ are the maximum traction and braking force at speed $v$, respectively.

Special utilization of the RE is usually not considered in minimizing the energy consumption of a single train, which means the energy model of single train only involves the traction energy, hence, the energy model of a train can be discretized by time step and defined as follow:

$$
E=\sum_{i=1}^{K}\left(F_{i} v_{i} / \eta_{t}\right) \Delta t .
$$

where $E$ denotes the traction energy of the train, $\eta_{t}$ denotes the conversion efficiency of traction system, $\Delta t$ is the simulation interval, and $K$ can be obtained by $K=T / \Delta t$.

\subsection{Train Group Model}

For the operation process of a train group, not only the traction energy of each train needs to be considered, but also the utilization of RE can't be ignored, which is generated by the braking train and can be recovered by the nearby traction trains. However, previous researchers 
mainly focus on a two-train system due to the complexity of multi-train cooperative control problem, actually, the total energy consumption of a $\mathrm{N}$-train $(\mathrm{N}>2)$ system can be defined as follow:

$$
E_{\text {sum }}=E_{1}+E_{2}+\cdots+E_{k}+\cdots+E_{N-1}+E_{N}-E_{R e_{-} s u m} .
$$

where $E_{k}(1 \leq \mathrm{k} \leq \mathrm{N})$ represents the traction energy of train $k$ and $E_{\text {Re sum }}$ represents the total recovered RE of the train group, which is expressed as follow, and $E_{R e_{-} i j}$ denotes the RE generated by train $i$ and recovered by train $j$.

$$
E_{\mathrm{Re}_{\_} \text {sum }}=\sum_{i=1}^{N} \sum_{j=1}^{N} E_{\mathrm{Re}_{-} i j} .
$$

Actually, total energy of a train group is hard to be minimized from above model, which can be transformed into the following equation:

$$
2 E_{\text {sum }}=E_{1}+\left[\left(E_{1}+E_{2}\right)+\cdots+\left(E_{k-1}+E_{k}\right)+\cdots\left(E_{N-1}+E_{N}\right)\right]+E_{N}-E_{R e_{-} s u m} .
$$

However, the total recovered RE $E_{\mathrm{Re} \text { sum }}$ involves the energy interaction of the train group, which still makes the multi-train collaboration problem difficult to solve. We arrange the train group by departure orders, it can be seen from above equation that, what if the RE of train $k-1(2 \leq k \leq N)$ is $100 \%$ absorbed by train $k$. In which situation, not only the total recovered RE is maximized, but also the complex distribution problem of $E_{\text {Re_sum }}$ is avoided, then the objective function for minimizing the train group energy consumption can be extended as follow:

$$
\begin{aligned}
& \min E_{\text {sum }}=\frac{1}{2}\left\{E_{1}+\left[\begin{array}{l}
\left(E_{1}+E_{2}-E_{R e_{-} \text {sum }}^{1}\right)+\cdots+\left(E_{k-1}+E_{k}-E_{R e_{-} \text {sum }}^{k-1}\right)+\left(E_{N-1}+E_{N}-E_{R e_{-} \text {sum }}^{N-1}\right) \\
\cdots+\left(E_{N}\right\} .
\end{array}\right]\right. \\
& \text { s.t. } \quad E_{R_{-} \text {sum }}=E_{R e_{-} \text {sum }}^{1}+\cdots+E_{R e_{-} \text {sum }}^{k-1}+\cdots+E_{R e_{-} \text {sum }}^{N-1} .
\end{aligned}
$$

where $E_{R e \_s u m}^{k}(1 \leq k \leq N-1)$ denotes the RE generated by train $k$. Therefore, train group collaboration problem can be turned into a single-train and multiple two-train problems, which can be optimized by departure orders, traction energy of the first train is minimized and speed profiles of rest trains are adjusted to maximize the utilization of RE by sequence. In other words, energy interaction only exists between adjacent trains, and the RE of each train just flows to its tracking train and won't influence the operation of other trains, energy flows in train group is shown in Fig. 1.

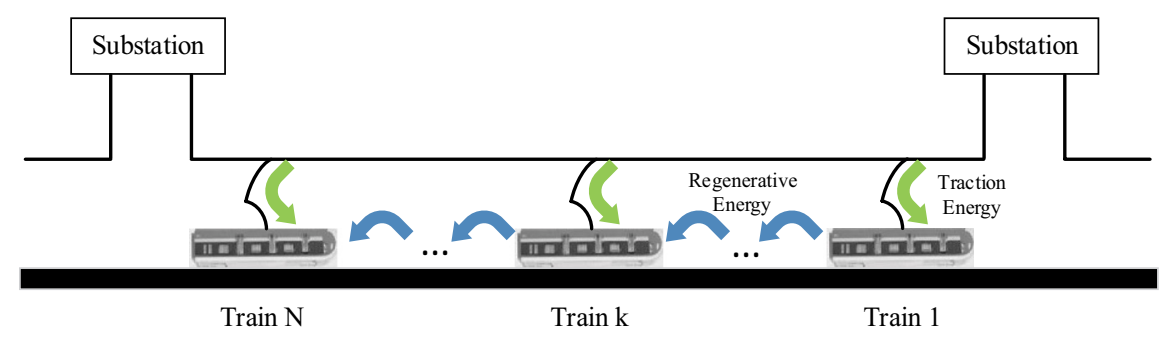

Figure 1: Energy flows in train group 


\subsection{Two Trains Model}

Taking the optimization of train $\mathrm{k}$ for example, to achieve the solution goal proposed above, a two-train system needs to be analysed, the energy model of which is expressed as follow:

$$
E_{\text {double }}^{k}=\left(E_{k-1}+E_{k}-E_{R e_{-} \text {sum }}^{k-1}\right) .
$$

where $E_{\text {double }}^{k}(2 \leq k \leq N)$ denotes the total energy of train $k$-1 and train $k$. Actually, speed profile of train $k-1$ has been optimized before optimizing train $k$, for which reason $E_{\mathrm{k}-1}$ can be considered as a constant here. Besides, optimization of train $k$ is independent, which only needs to focus on the braking power of train $k-1$, then the speed profile of train $k$ is adjusted to $100 \%$ recover the RE generated by train $k-1$, because the RE generated by other previous trains have been fully absorbed. Based on above analysis, the energy model of two-train system can be transferred as follow:

$$
E_{\text {double }}^{k}=\sum_{i=1}^{M}\left(F_{i}^{k} v_{i}^{k} / \eta_{t}-P_{i}^{k-1}\right) \Delta t+C_{k-1} .
$$

here, $P_{i}^{k-1}$ represents the braking power of train $k-1$ at simulation step $i$, and $C_{k-1}$ represents the traction energy of train $k-1$ which is a constant.

In the braking process of train $k$-1, appropriate traction force of train $k$ at simulation step $i$ can be taken to fully recover the RE, which is decided by the braking power of train $k-1$ and can be deduced from above equation and shown as follow:

$$
F_{i}^{k}=\eta_{t} P_{i}^{k-1} / v_{i}^{k}
$$

\section{SOLUTION METHODS}

\subsection{Optimization for Single Train}

Based on the analysis of above models, train group collaboration problem is turned into a single-train and multiple two-train problems, since the first train has no RE to absorb, the traction energy of which needs to be minimized before optimizing the rest trains.

For metro system, the distances between stations are very short, in which situation the optimal control strategy of a single train is maximum traction (MT), coasting (CO) and maximum braking (MB) by sequence [15]. Therefore, optimizing the speed profile of single train means to find a trajectory consisting of MT, $\mathrm{CO}$ and $\mathrm{MB}$ in order that satisfies both trip time and distance requirements, detailed solution method is shown in Fig. 2.

The acceleration curve from 0 to the speed limit $V$ with MT is calculated, then we search back from the last point, in which the train switches from MT to CO and stops at time $T$ with $\mathrm{MB}$, until the area surrounded by the three phases speed profile is equal to the distance of the subsection, namely both trip time and distance constraint are met, and the optimal trajectory of a single-train is obtained. 


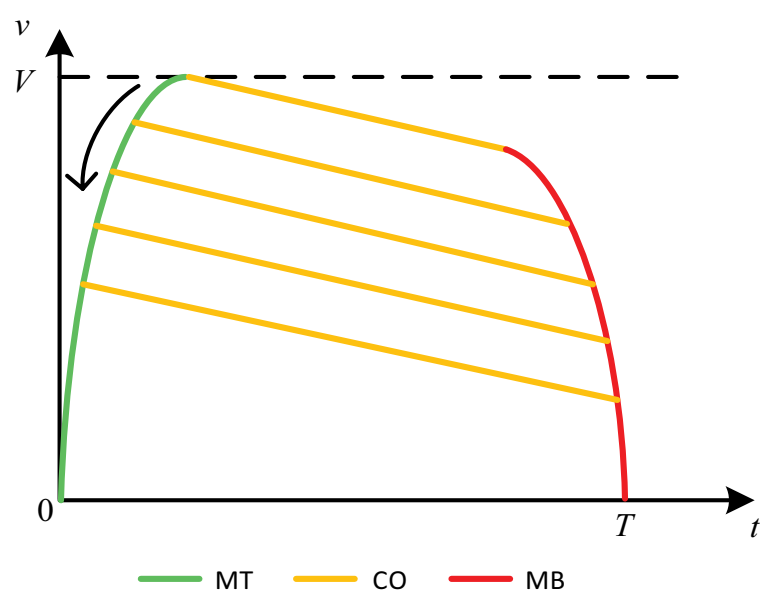

Figure 2: Optimization method of the first train

\subsection{Optimization for Train Group}

Basic idea for train group optimization is that the speed profile of each train is adjusted to fully absorbed the RE generated by its previous train until Train $N$ is optimized, as mentioned before, speed profiles of rest trains can be optimized by departure orders after the traction energy of the first train is minimized, which is shown in Fig. 3.

The advantage of this approach can be easily observed from above picture, $100 \%$ RE utilization of previous train for each train means that the total power curve of optimized multitrain system is very simple, which always leaves just one segment of braking power for the next train as shown in Fig. 4, and makes the optimization of each train independent. Taking the optimization of Train $k$ as an elaboration, which only needs to focus on the braking power of Train $k-1$ and the speed profiles of other previous $k$ - 2 trains can be ignored, because the RE of Train 1 to Train $k-2$ have all been recovered, thus cleverly avoiding the complex cooperation of train group.

Based on above analysis, the key to obtain the optimal speed profiles of train group is to find a way to adjust the trajectory of a train to fully absorb the RE according to a given braking power segment. Combining the proposed two-train system model, appropriate traction force in each simulation step must be taken during the RE absorption process, which is time-varying and determined by the braking power of the previous train, besides, operation constraints should be also met, detailed solution method is shown in Fig. 5.

Assume the departure time of Train $k$ is $t_{k}$, the start and end time of the braking segment of Train $k-1$ are $t_{k}^{1}$ and $t_{k}^{2}$, respectively. The first step of the solution method is same as the optimization of a single train, namely the acceleration curve from 0 to the speed limit with MT is calculated, then the searching process starts at the last point and goes backward, from which the train replaces MT with $\mathrm{CO}$, until the trip time arrives at $t_{k}^{1}$, where the RE absorption process begins. As the speed $v_{k}^{1}$ is known at time $t_{k}^{1}$, so as corresponding braking power of Train $k-1$, from both of which the optimal traction force can be calculated by equation (17) to fully absorb the braking power, hence, the traction force in each step from $t_{k}^{1}$ to $t_{k}^{2}$ can be obtained the same way, thus calculating $v_{k}^{2}$ is simple, which is the speed at time $t_{k}^{2}$. RE of previous train has been fully recovered until now, then the train takes 


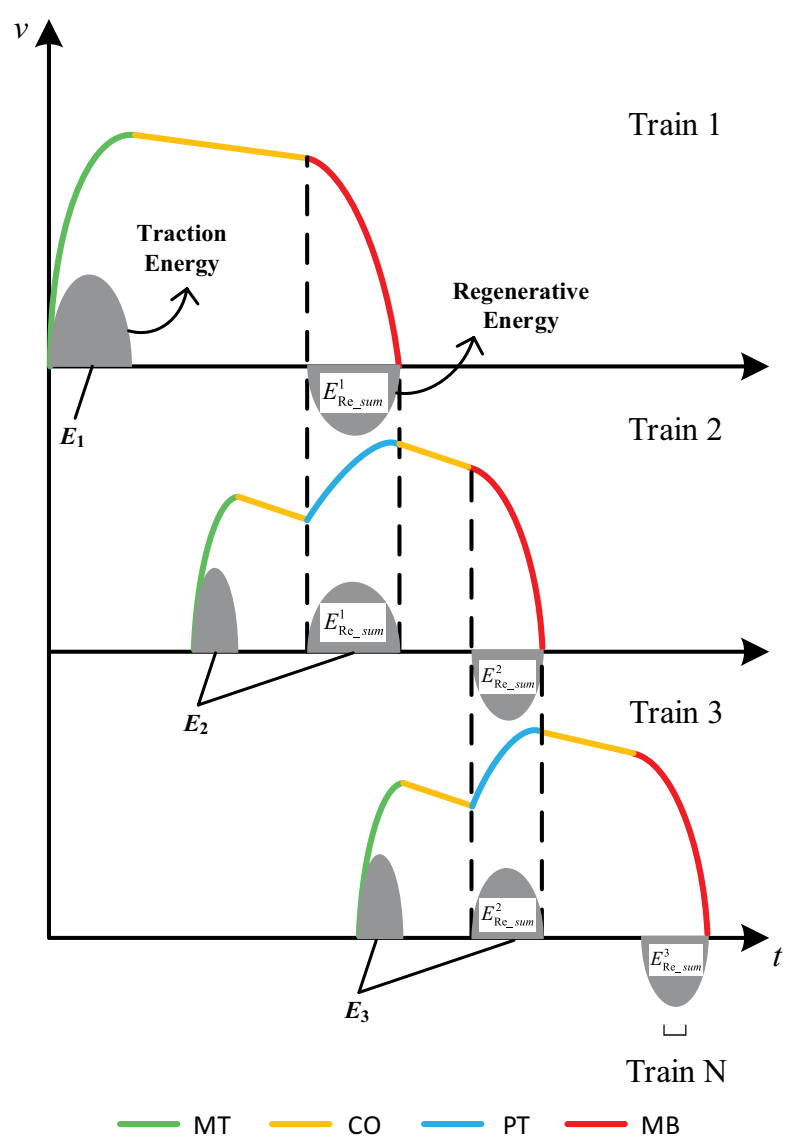

Figure 3: Trajectory optimization of train group

CO mode and stops at time $t_{k}+T$ with MB. Searching process will not stop until the area surrounded by the optimized speed profile is equal to the distance of the subsection, namely both trip time and distance constraint are met, then the RE of Train $k-1$ is $100 \%$ recovered and the optimal speed profile of Train $k$ is obtained.

It's important to note here that, in some search points at the beginning, cruising (CR) mode may appears at the absorption phase when the speed accelerates to speed limit for safety, in which situation the utilization of RE in CR phase may not reach $100 \%$. Normally the control strategy during $\left[t_{k}^{1}, t_{k}^{2}\right]$ of the optimal speed profiles are partial traction (PT), because the traction force determined by the braking power at high-speed area usually won't exceed the maximum.

\section{CASE STUDY}

In this section, simulation and comparative results based on Guangzhou Metro Line 8 are presented to verify the effectiveness and validity of proposed optimization methods. We select one subsection from Chigang Station to Kecun Station for experiment, the distance and planned trip time of which are $1489 \mathrm{~m}$ and $97 \mathrm{~s}$, respectively. Besides, the amount of the train group is 3 with a given departure time set $\left[\Delta T_{1}, \Delta T_{2}\right]$. Detailed operation parameters are shown in Table 1 and the line conditions are shown in Table 2. 


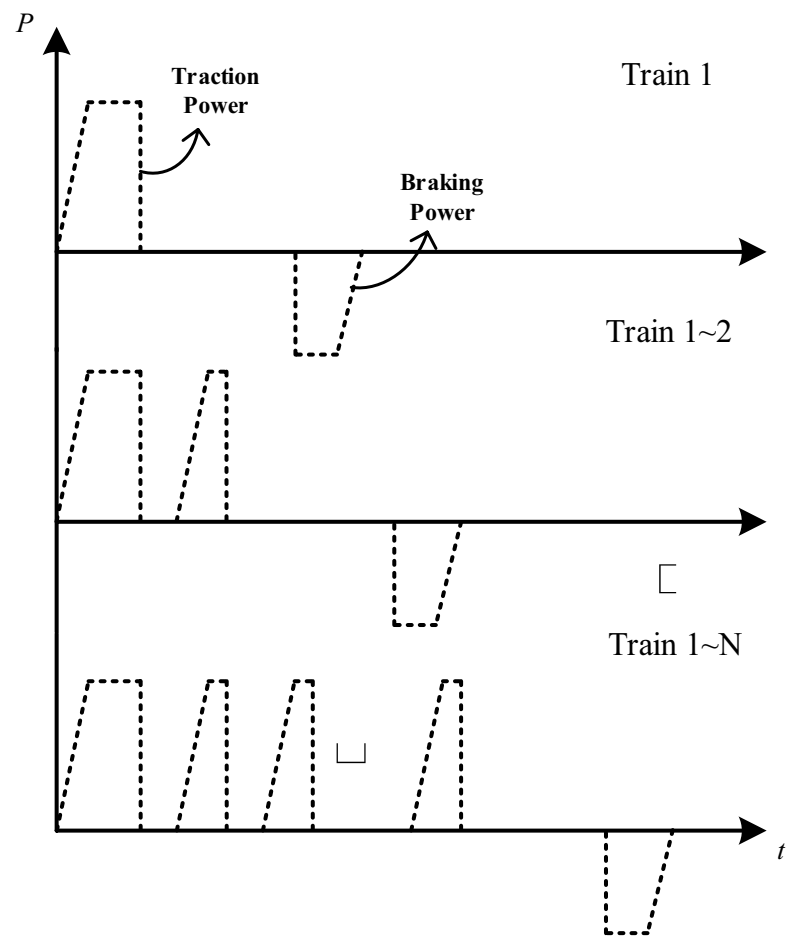

Figure 4: Total power curves of multi-train

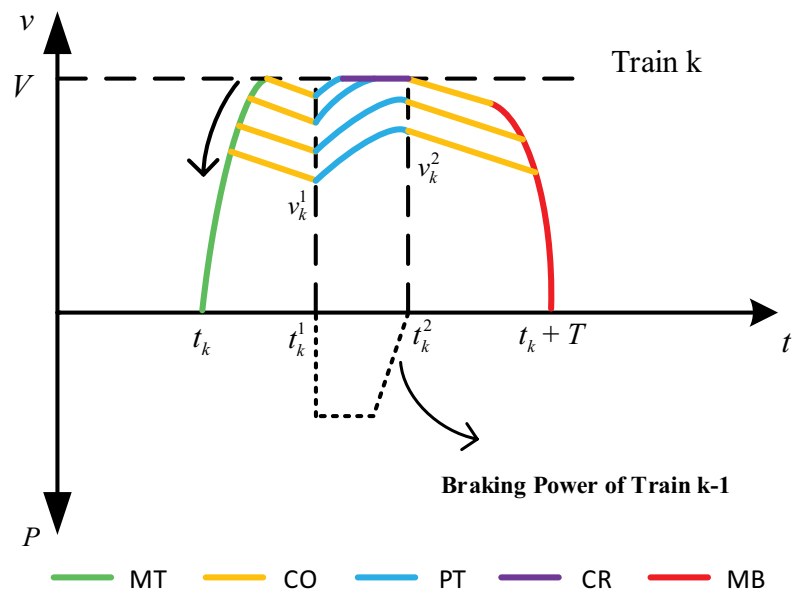

Figure 5: Optimization method of Train $\mathrm{k}$ 
Table 1: Operation parameters

\begin{tabular}{llllll}
\hline Parameters & $M / t$ & $\eta_{\mathrm{t}}$ & $\Delta T_{1} / s$ & $\Delta T_{2} / s$ & $\Delta t / s$ \\
Value & 236.98 & $90 \%$ & 35 & 30 & 0.1 \\
\hline
\end{tabular}

Table 2: Line conditions

\begin{tabular}{lll}
\hline Segments $(\mathrm{m})$ & Gradient $(\% \circ)$ & Speed Limit $(\mathrm{km} / \mathrm{h})$ \\
\hline$[3732,3794)$ & -3 & 80 \\
{$[3794,4075)$} & -3 & 65 \\
{$[4075,4163)$} & -3 & 80 \\
{$[4163,4199)$} & -3 & 75 \\
{$[4199,4361)$} & -4 & 75 \\
{$[4361,4489)$} & -4 & 80 \\
{$[4489,5099)$} & 9.636 & 80 \\
{$[5099,5221]$} & 3 & 80 \\
\hline
\end{tabular}

Besides, the characteristic of basic running resistance $R_{1}(v)$, maximum traction/braking force $F_{t}^{\max }(v)$ and $F_{b}^{\max }(v)$ are given as follow:

$$
\begin{gathered}
R_{1}(v)=9.067+1.334 \times 10^{-3} v^{2} . \\
F_{t}^{\max }(v)= \begin{cases}400 & 0 \leq v \leq 40 \mathrm{~km} / \mathrm{h} \\
16000 / v & 40 \leq v \leq 55 \mathrm{~km} / \mathrm{h} \\
880000 / v^{2} & 55 \leq v \leq 80 \mathrm{~km} / \mathrm{h}\end{cases} \\
F_{b}^{\max }(v)= \begin{cases}0 & 0 \leq v \leq 3 \mathrm{~km} / \mathrm{h} \\
194.5(v-3) & 3 \leq v \leq 5 \mathrm{~km} / \mathrm{h} \\
389 & 5 \leq v \leq 48.5 \mathrm{~km} / \mathrm{h} \\
913962.5 / \mathrm{v}^{2} & 48.5 \leq v \leq 80 \mathrm{~km} / \mathrm{h}\end{cases}
\end{gathered}
$$

Based on the proposed single-train optimization method, traction energy of the first train is minimized and the optimized trajectory is shown in Fig. 6, so as the practical speed profile. Traction energy of the two speed profiles is shown in Table 3, compared to practical operation, energy saving rate by single-train optimization can reach up to $11.21 \%$. 


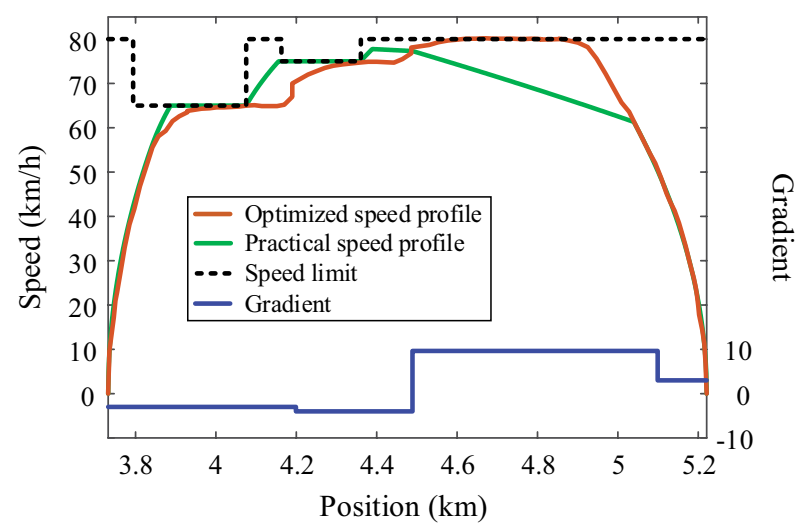

Figure 6: Optimized speed profile of Train 1

Table 3: Traction energy of two speed profiles

\begin{tabular}{ll}
\hline Speed profile & Traction energy $(\mathrm{kWh})$ \\
\hline Practical & 20.08 \\
Optimized & 17.83 \\
\hline
\end{tabular}

For most existing metro systems, specific utilization of RE is usually not taken into consideration in practical operation, therefore, we firstly let each train of train group takes the same trajectory with the first train, which means total traction energy of the train group in minimized without considering the utilization of RE, speed profiles and power curves of the train group are shown in Fig. 7. It can be seen from the power curve that the RE of train group can't be effectively recovered, actually, it totally depends on the given departure times since the control strategy of each train are fixed.

Energy interaction plays an important role in energy saving of train group operation, which means that minimizing traction energy usually can't minimise total energy. With considering specific utilization of RE, the trajectory of train group is jointly optimized by proposed method to maximize the utilization of RE, optimized speed profiles and power curves of the train group are shown in Fig. 8. The first shaded area represents the braking process of Train 1, during which Train 2 absorbs the RE of Train 1 by accelerating, and the traction force of Train 2 in this area is decided by braking power of Train 1 to fully recover RE. It is easy to observe from the power curve that RE of Train 1 is $100 \%$ recovered by Train 2, achieving the effect described in Fig. 4, only the braking power of Train 2 left in the optimization of Trian 3 , which won't be influenced by the operation of Train 1. Same for the second shaded area, Train 3 accelerates to absorb the RE of Train 2, however, total power curve shows that the RE recovery failed to reach $100 \%$ this time, which can be known from the deep part of this area that, the speed of Train 3 reaches the limit and only needs to keep cruising for safety, meanwhile, the traction power in keeping speed is not enough to recover the RE, making a small amount of RE has not been absorbed. 
(a)

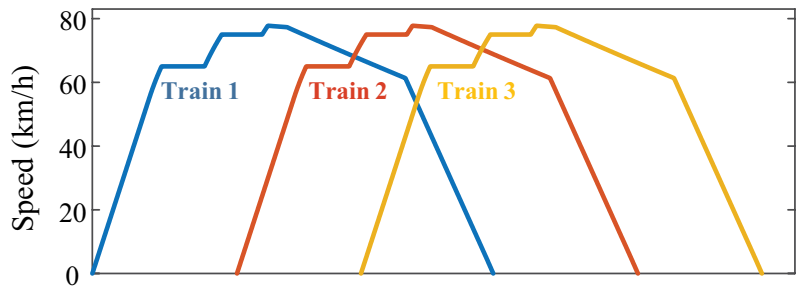

(b)

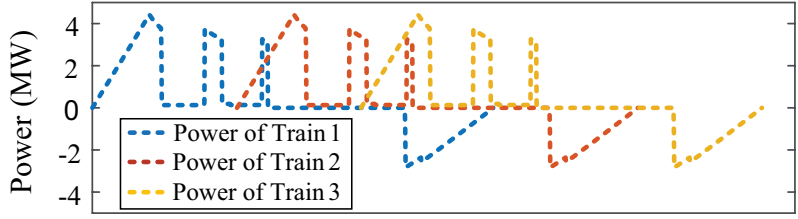

(c)

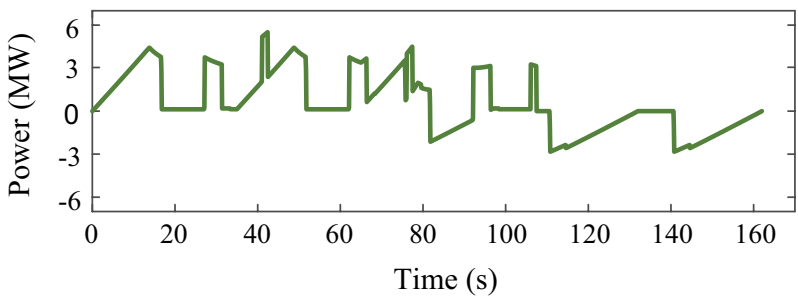

Figure 7: Operation curves of train group before joint optimization. (a) Speed profiles of each train; (b) Power curves of each train; (c) Total power curve of train group.

(a)

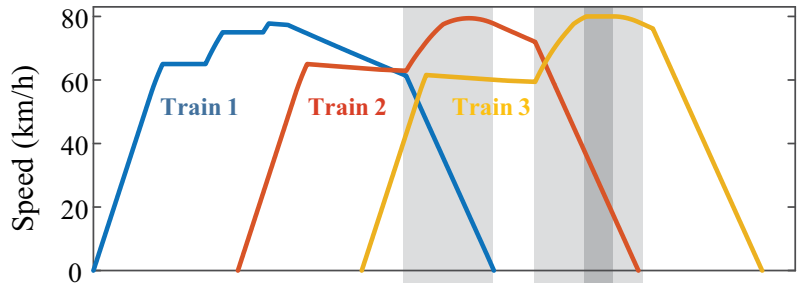

(b)

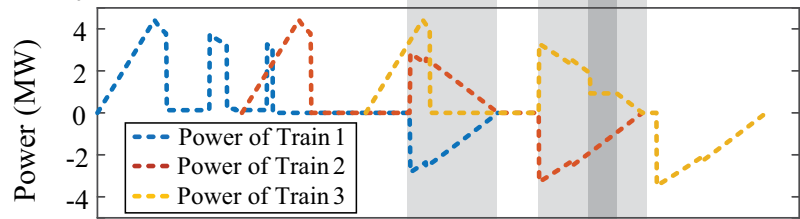

(c)

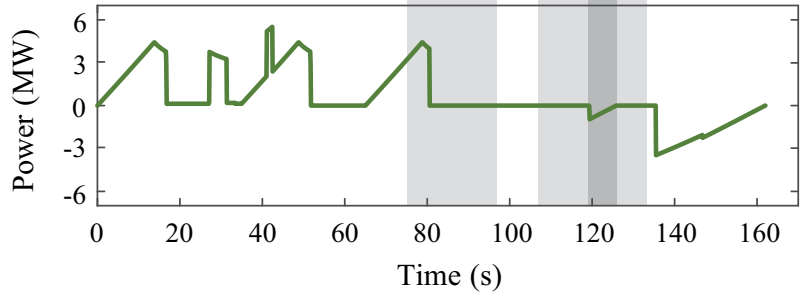

Figure 8: Operation curves of train group after joint optimization. (a) Speed profiles of each train; (b) Power curves of each train; (c) Total power curve of train group. 
Table 4: Energy consumption before and after optimization of train group

\begin{tabular}{lllcll}
\hline Case & $\begin{array}{l}\text { Traction } \\
\text { energy }(\mathrm{kWh})\end{array}$ & $\begin{array}{l}\text { Generated } \\
\text { RE }(\mathrm{kWh})\end{array}$ & $\begin{array}{l}\text { Recovered } \\
\text { RE }(\mathrm{kWh})\end{array}$ & $\begin{array}{l}\text { Total energy } \\
(\mathrm{kWh})\end{array}$ & $\begin{array}{l}\text { Utilization } \\
\text { of RE }\end{array}$ \\
\hline Fig. 7 & 53.49 & 18.20 & 5.12 & 48.37 & $28.13 \%$ \\
Fig. 8 & 60.55 & 21.35 & 20.49 & 40.06 & $95.97 \%$ \\
\hline
\end{tabular}

Comparison of detailed energy consumption of the two control modes of train group mentioned above is shown in Table 4, compared to the minimum traction energy cost mode, the energy saving rate by joint optimization of train group achieves $17.18 \%$. Although the adjustment of trajectory of trains leads to an apparent increase in traction energy, it reaches a high utilization of RE and minimizes the total energy of train group. Without considering the RE of the last train, which has no other train to absorb in this case, the utilization of RE of train group reaches $95.97 \%$, although it doesn't reach $100 \%$ due to the safety operation constraint, it is very close, and the effective use of RE significantly reduces the total energy consumption of train group. Besides, our method still works as the number of trains increases.

\section{CONCLUSION}

In this paper, energy model of train group is analyzed, a novel solution method is proposed by turning the complex multi-train cooperation problem into a single-train and multiple twotrain problems. Then the optimal traction force determined by the braking power during the RE absorption process is deduced to fully recover the RE in a two-train system, which enables the train group to be optimized by departure orders, namely the traction energy of the first train is minimized and the speed profile of rest trains are adjusted by sequence to maximize the utilization of RE. Optimization of each train is independent and complex multitrain collaborative problem is greatly simplified. Detailed optimization methods are proposed and the effectiveness is verified by the simulation results based on Guangzhou Metro. Train group optimization has huge energy saving space, which is especially of great significant for practical engineering applications in Metro system.

\section{REFERENCES}

[1] Gonzlez-Gil, A., Palacin, R., Batty, P. \& Powell, J., A systems approach to reduce urban rail energy consumption. Energy convers. Manag, 80, pp. 509-524, 2014.

[2] Peter, P. \& Howlett, P., Optimal driving strategies for a train journey with speed limits. Anziam. J, 36, pp. 38-49, 1994.

[3] Howlett, P. \& Cheng, J., Optimal driving strategies for a train on a track with continuously varying gradient. Bulletin Of The Australian Mathematical Society, 38, pp. 388-410, 1997.

[4] Khmelnitsky, E., On an optimal control problem of train operation. IEEE Transactions on Automatic Control, 45(7), pp. 1257-1266, 2000.

[5] Liu, R. \& Golovitcher, I.M., Energy-efficient operation of rail vehicles. Transportation Research Part A-Policy and Practice, 37(10), pp. 917-932, 2003.

[6] Albrecht, A., Howlett, P., Pudney, P. \& Vu, X., Energy-efficient train control: From local convexity to global optimization and uniqueness. Automatica, 49(10), pp. 3072-3078, 2013. 
[7] Shuai, S., Xiang, L., Tao, T. \& Ziyou, G., A Subway Train Timetable Optimization Approach Based on Energy-Efficient Operation Strategy. IEEE Transactions on Intelligent Transportation Systems, 14(2), pp. 883-893, 2013.

[8] Ramos, A., Pena, M.T., Fernández, A. \& Cucala, P., Mathematical programming approach to underground timetabling problem for maximizing time synchronization. Management and Organization, 35, pp. 88-95, 2008.

[9] Scheepmaker, G.M., Goverde, R.M.P. \& Kroon, L.G., Review of energy-efficient train control and timetabling. European Journal of Operational Research, 257, pp. 355-376, 2017.

[10] Shuai, S., Tao, T. \& Roberts, C., A Cooperative Train Control Model for Energy Saving. IEEE Transactions on Intelligent Transportation Systems, 16(2), pp. 622-631, 2015.

[11] Xun, J., Tao, T., Song, X., Wang, b. \& Jia, z., Research on energy-saving driving model with regenerative energy considered. China Railway Soc, 36(1), pp. 104-149, 2015.

[12] Haichuan, T., Dick, C.T. \& Xiaoyun, F., Improving Regenerative Energy Receptivity in Metro Transit Systems. Transportation Research Record: Journal of the Transportation Research Board, 2534, pp. 48-56, 2015.

[13] Jianqiang, L., Huailong, Guo. \& Yinxue, Y., Research on the Cooperative Train Control Strategy to Reduce Energy Consumption, IEEE Transactions on Intelligent Transportation Systems, 18(5), pp. 1134-1142, 2017.

[14] Mo, C., Zhuang, X., Pengfei, S., Qingyuan, W., Bo, J. \& Xiaoyun, F., Energy-Efficient Driving Strategies for Multi-Train by Optimization and Update Speed Profiles Considering Transmission Losses of Regenerative Energy. Energies, 12, pp. 3573, 2019.

[15] Horst, S., Peter, H. \& Manfred, K., Contribution to Optimum Computer-Aided Control of Train Operation. Proceedings of the Second IFAC/IFIP/IFORS Conference on Control in Transportation Systems, pp. 377-385, 1974. 